\title{
Encoding Dependency Information inside Tree Transformer
}

\author{
Mahtab Ahmed ${ }^{\dagger}, *$, Robert E. Mercer ${ }^{\dagger}$ \\ $\dagger$ The University of Western Ontario
}

\begin{abstract}
Representing a sentence in a high dimensional space is fundamental for most natural language processing (NLP) tasks at present. These representations depend on the underlying structures upon which they are built. Two scenarios are possible: one is to view the sentence as a sequence of words and another is to consider its inherent grammatical structure. It is possible to equip the first way with some external grammatical knowledge, but to capture a proper syntax would be close to impossible. Therefore, we investigate the second one by extending the design of an existing dependency tree transformer (DT-Transformer). We propose adding a novel edge encoding mechanism to this prior architecture. Experiments show that in sentence encoding, having access to information about the relationships between "head" words and their "dependent" words and how the heads are influenced by the dependent words achieves better sentence representation. Evaluation on the four tasks shows noteworthy results compared to the existing DT-Transformer, standard Transformer, LSTM-based models, and treestructured LSTMs. Extensive experimentation with representing the edge embeddings as different distributions (mean and standard deviation), encoding the edges in different ways, and an ablation study to find where to place each module in the architecture and which modules to use in the design is also provided.
\end{abstract}

Keywords: Tree Transformer, Multi-head attention, Multi-branch attention, Natural language inference, Dependency parsing, and Paraphrase detection.

\section{Introduction}

Following the breakthrough in natural language processing (NLP) research with word embeddings [1], recent research has had its focus shifted to developing effective sentence embeddings [2, 3]. Having good generalized sentence representations is fundamental for solving a range of NLP tasks because they eventually involve sentences, e.g., question answering, sentiment analysis, semantic similarity, and natural language inference. Encoding a sentence requires word level information either as a sequence or in a structured form. Large scale models can afford to overlook sentence structure as they deal with lots of data and have access to large computing power for training [4-6], whereas on a smaller scale, having access to structural information gives a great boost even when training with less data.

In traditional linguistics, dependency parses are used to represent the relationships among words as triples of a relation between pairs of words $[7,8]$. In this paper, we propose a novel edge encoding mechanism of a dependency parse tree and extend the design of one of the existing dependency tree transformer models [9] using very few extra parameters. To the best of our knowledge, no work has been done on encoding these head-dependent relations into a dependency tree edge. We share the same composition function across word level encoding and edge level encoding, allowing the attention module to transfer knowledge across these two levels. Unlike existing tree structured models [10, 11], the number of parameters in our model is not dependent on the number of dependents under a head node. We present visualizations showing how our model makes a classification decision by observing the attention probabilities in the tree. Our extensive evaluation shows that the edge label encoding information certainly helps to improve our model or to be at least on

*mahme255@uwo.ca

This article is () 2021 by author(s) as listed above. The article is licensed under a Creative Commons Attribution (CC BY 4.0) International license (https://creativecommons.org/licenses/by/4.0/legalcode), except where otherwise indicated with respect to particular material included in the article. The article should be attributed to the author(s) identified above. 
par compared to the existing sequential (i.e., LSTM and Transformer) and tree-structured (i.e., Tree LSTM, RvNN and Tree Transformer) models.

\section{Related Work}

Most recent sentence encoders utilize deep learning-based encoding to obtain a dense semantic representation. These encoders treat the sentence either in the raw form just as a sequence of words or look at its tree representation generated from a dependency or constituency parser. We will summarize prior work related to the modules of our proposed model and highlight existing architectures for natural language inference, paraphrasing and sentiment analysis tasks.

The attention mechanism adopted by most of the RNN-based models requires access to the hidden states at every time step $[12,13]$. This introduces a wait time as all hidden states have to be generated prior, making it hard to parallelize. To overcome this, Parikh, Täckström, Das, and Uszkoreit [14] propose a query and key-based fully attentive neural network which depends on matrix multiplication to calculate the attention probability and is easily parallelizable. Later, Vaswani, Shazeer, Parmar, Uszkoreit, Jones, Gomez, Kaiser, and Polosukhin [15] adopt this and build a state of the art machine translation model called Transformer. The encoder portion of transformer operates using the multi-head version of the attention module. Following this, Ahmed, Keskar, and Socher [16] propose a multibranch version of transformer where they claim that it is important to allow different heads to operate to their required extent rather than to make all of them contribute uniformly. Cer, Yang, Kong, Hua, Limtiacob, John, Constant, Guajardo-Céspedes, Yuanc, Tar, Sung, Strope, and Kurzweil [3] propose an unsupervised sentence encoder by leveraging only the encoder part of Transformer. They train on the large Stanford Natural Language Inference (SNLI) corpus and then use transfer learning on smaller task-specific corpora.

Large scale language models have proven to be very effective in providing good sentence representations. Devlin, Chang, Lee, and Toutanova [4] propose a general-purpose NLP architecture by stacking several transformer encoders where a special token padded at the beginning of the sentence contains the sentence representation. Radford, Narasimhan, Salimans, and Sutskever [17] uses multilayer transformer decoders where multi-head selfattention is applied over the input followed by a softmax to get the output distribution over the target tokens. In this series, Lewis, Liu, Goyal, Ghazvininejad, Mohamed, Levy, Stoyanov, and Zettlemoyer [5] combines the prior two methods and proposes a machine translation model where the former one reconstructs the [MASK] tokens at the encoder and the latter one performs an auto-regressive prediction at the decoder.

Incorporating tree structured linguistic information has been seen to improve the RNNbased models. Socher, Lin, Ng, and Manning [10], Socher, Karpathy, Le, Manning, and Ng [11], and Socher, Perelygin, Wu, Chuang, Manning, Ng, and Potts [18] propose a family of recursive neural net (RvNN) based models, where a composition function is applied recursively bottom-up on child nodes to compute the parent node representation until the root is reached. Tai, Socher, and Manning [19] propose two tree-structured LSTMs, where the hidden and cell state of a parent node depends only on its children, allowing the connection between a head and its dependent (dependency Tree LSTM) or on the words within a phrase (constituency tree LSTM) to remain intact. Ahmed, Samee, and Mercer [20] further encodes attention inside these tree version LSTMs showing the degree to which different children contribute to generate the parent node representation. Ahmed, Samee, and Mercer [9] propose a tree version of transformer where they replace the composition function with an attention module and test it on four different NLP tasks. 


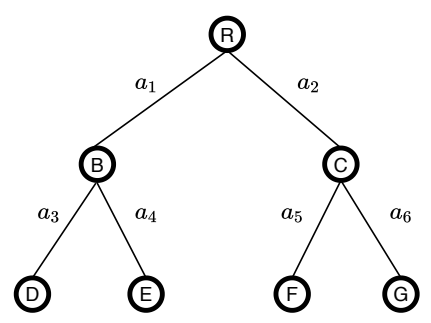

(a) Tree to traverse

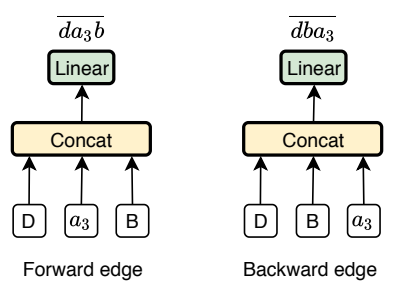

(b) Relationship representation

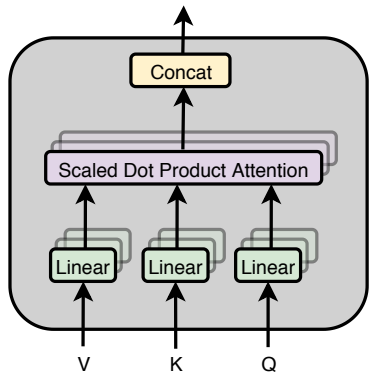

(c) Attention block used

Figure 1. Design Components.

\section{Model}

In this section, we describe the model in detail. We are motivated to traverse a dependency tree using the attention module designed for a standard Transformer. Unlike the tree transformer [9] that we are extending, we leverage both word and edge information. First, we describe the design components which include a novel edge encoding mechanism followed by an in-depth exposure to the general architecture of our edge encoded Tree Transformer model. Finally, we conclude this section by suggesting some task-specific modifications.

\subsection{Design Components}

We start by first extracting the dependency tree of a given sentence using the Stanford CoreNLP 3.9.1 parser with Universal Dependencies version 1 [21] as shown in Figure 1(a). A dependency tree has word information $(\mathbf{R} \rightarrow\{B, C\} ; \mathbf{B} \rightarrow\{D, E\} ; \mathbf{C} \rightarrow\{F, G\})$ on every node and we represent this information with word vectors $\mathbf{X} \in \mathbb{R}^{1 \times d}$ initialized by some standard pre-trained word embeddings. In addition to this, each edge is marked with dependency information of which no standard representation is available. We initialize an edge embedding layer with a normal distribution having a mean $(\mu)$ and a standard deviation $(\sigma)$ to represent the $N$ unique dependency labels from the Stanford parser [7].

$$
\mathbf{a}_{\mathbf{i}} \in \mathcal{N}(\mu, \sigma) ; \forall \mathbf{i} \in[1 \ldots N]
$$

We propose a novel encoding mechanism of the dependency tree edges as shown in Figure 1(b). It is to be noted that there exist other encoding mechanisms but this one gives comparatively better results. Paper length does not allow presentation of all possible combinations. We hypothesize that representing an edge in two ways (we call them forward and backward) conveys more information than representing in just one way. Linguistically, it represents a word being modified by other words and a word modifying other words. Each edge has three participants: parent $(\mathbf{q})$, child $(\mathbf{x})$ and edge label $(\mathbf{y})$. Our extensive experiments indicate that $\mathbf{q}, \mathbf{x}$, and $\mathbf{y}$ can be in any permutation as long as the orders for forward (f) and backward (b) are unique. We create an edge representation by concatenating the participants and create two large vectors as follows,

$$
\mathbf{f}^{1 \times 3 d}=[\mathbf{q} ; \mathbf{y} ; \mathbf{x}] \quad \mathbf{b}^{1 \times 3 d}=[\mathbf{q} ; \mathbf{x} ; \mathbf{y}]
$$

Next, we project these edge representations into a lower dimension $d$ just to be consistent with the dimension of the word embedding as follows,

$$
\overline{\mathbf{f}}^{1 \times d}=\mathbf{W}_{\mathbf{1}} \mathbf{f} \quad \overline{\mathbf{b}}^{1 \times d}=\mathbf{W}_{\mathbf{2}} \mathbf{b}
$$


We use multi-head self-attention [15] as the composition function of a sub-tree as shown in Figure 1(c). It starts by projecting the input into a lower dimension $n$ times ( $n$ being the number of heads) and applies a self-attention (SA) block on top of them. This SA block is equipped with three projection matrices: $\mathbf{W}_{Q}, \mathbf{W}_{K}$ and $\mathbf{W}_{V}$. It considers the input tensor as query, key, and value matrices. First, it transforms the query and key matrices as $\mathbf{W}_{Q} Q$ and $\mathbf{W}_{K} K$. Next, it uses a dot-product for the alignment followed by softmax to get the attention probabilities. Finally, it multiplies these probabilities with the transformed value matrix $\mathbf{W}_{V} V$ to get a scaled representation. All of these computations are summarized in Eqn. 3.4. The $\mathbf{p}_{\mathbf{i}}$ 's are concatenated to get the final attentive representation.

$$
\mathbf{p}_{\mathbf{i}}=\operatorname{softmax}\left(\frac{\left(\mathbf{W}_{Q} Q\right)\left(\mathbf{W}_{K} K\right)^{T}}{\sqrt{\mathbf{d}_{k}}}\right)\left(\mathbf{W}_{V} V\right)
$$

\subsection{General Architecture}

As shown in Figure 2, our general architecture involves traversing the tree twice: once using the word information and another using the edge label information. It is to be noted that each traversal of a subtree is represented by an encoder block which takes as many children as it has for input (i.e., in the leftmost leaf $\overline{d a_{3} b}, \overline{e a_{4} b}$ for forward edge encoding and right next to it $\overline{d b a_{3}}, \overline{e b a_{4}}$ for backward edge encoding). However, the composition function $(\mathbf{F})$ remains the same for both cases. We now explain what is used as input to this composition function for both cases. Later in this subsection we explain how we can extract the final parent representation utilizing the transformed input.

Word Traversal: Dependency trees contain a word at every node. To traverse a subtree in a dependency tree, we look at both the parent and child representations by concatenating them as a tensor $\mathbf{u}$ as follows,

$$
\mathbf{u}=\left[\mathbf{q} ; \mathbf{x}_{\mathbf{1}} ; \ldots ; \mathbf{x}_{\mathbf{n}}\right]
$$

Here, $\mathbf{q}$ is the parent representation and the $\mathbf{x}_{i}$ 's are the child representations. Finally, we apply $\mathbf{F}$ over $\mathbf{u}$ to get the attentive transformed representation of a subtree as $\mathbf{p}_{\mathbf{u}}=\mathbf{F}(\mathbf{u})$. Edge Traversal: Given a subtree, we traverse it in two different ways: one with forward edges and one with backward edges. We create two new tensors $\mathbf{v}^{\mathbf{f}}$ and $\mathbf{v}^{\mathbf{b}}$ representing the forward and backward edge versions, respectively,

$$
\begin{array}{r}
\mathbf{v}^{\mathbf{f}}=\left[\overline{\mathbf{f}_{\mathbf{1}}} ; \overline{\mathbf{f}_{2}} ; \ldots ; \overline{\mathbf{f}_{n}}\right] \\
\mathbf{v}^{\mathbf{b}}=\left[\overline{\mathbf{b}_{1}} ; \overline{\mathbf{b}_{2}} ; \ldots ; \overline{\mathbf{b}_{n}}\right]
\end{array}
$$

Here, the f's and b's are the forward and backward edge representations as defined above. Finally, we apply the composition function to them as follows,

$$
\mathbf{p}_{\mathbf{v}^{\mathbf{f}}}=\mathbf{F}\left(\mathbf{v}^{\mathbf{f}}\right) \quad \mathbf{p}_{\mathbf{v}^{\mathbf{b}}}=\mathbf{F}\left(\mathbf{v}^{\mathbf{b}}\right)
$$

Parent Representation: As mentioned before, we use a multi-head self-attention block as the composition function (F) [15]. For each of the representations, $\mathbf{u}, \mathbf{v}^{\mathbf{f}}$ and $\mathbf{v}^{\mathbf{b}}$, after transforming the inputs using this $\mathbf{F}$, we apply a residual connection, transform it non-linearly and perform an element-wise summation $(\mathrm{EwS})$ to get the three hidden representations of the subtree. The computations for word traversal and edge traversal are shown in Eqn. 3.9.

$$
\begin{aligned}
\tilde{\mathbf{p}}_{\mathbf{u}} & =\tanh \left(\operatorname{EwS}\left(\left(\mathbf{p}_{\mathbf{u}}+\mathbf{u}\right) \mathbf{W}+b\right)\right) \\
\tilde{\mathbf{p}}_{\mathbf{v}^{\mathbf{f}}} & =\tanh \left(\operatorname{EwS}\left(\left(\mathbf{p}_{\mathbf{v}^{\mathbf{f}}}+\mathbf{v}^{\mathbf{f}}\right) \mathbf{W}+b\right)\right) \\
\tilde{\mathbf{p}}_{\mathbf{v}^{\mathbf{b}}} & =\tanh \left(\operatorname{EwS}\left(\left(\mathbf{p}_{\mathbf{v}^{\mathbf{b}}}+\mathbf{v}^{\mathbf{b}}\right) \mathbf{W}+b\right)\right) \\
\tilde{\mathbf{p}}_{\mathbf{v}} & =\tilde{\mathbf{p}}_{\mathbf{v}^{\mathbf{f}}}+\tilde{\mathbf{p}}_{\mathbf{v}^{\mathbf{b}}}
\end{aligned}
$$




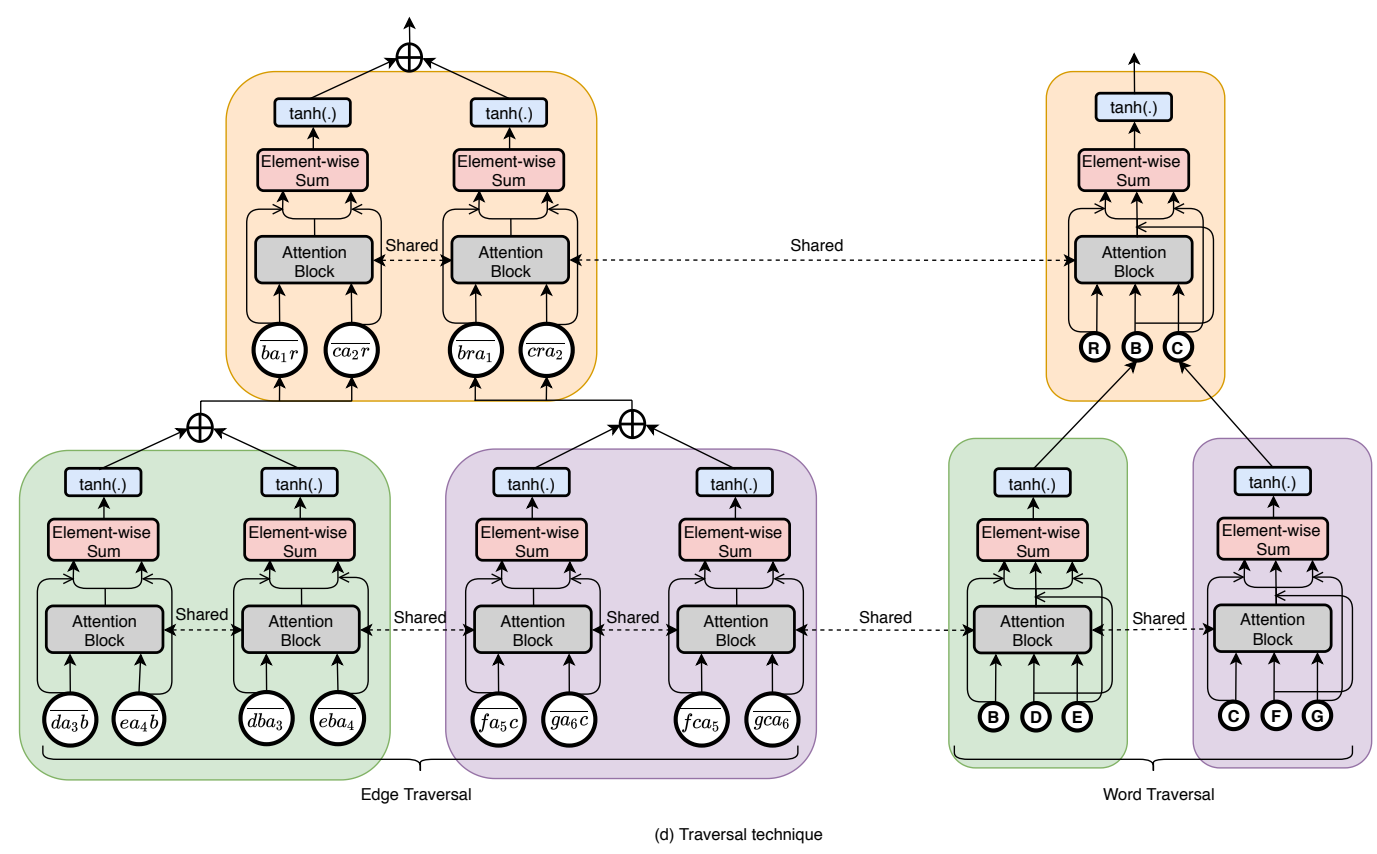

Figure 2. Encoding edge information inside dependency tree transformer.

Finally, at the root, we concatenate $\tilde{\mathbf{p}}_{\mathbf{u}}$ and $\tilde{\mathbf{p}}_{\mathbf{v}}$ to get the final tree representation $(R=$ $\left.\left[\tilde{\mathbf{p}}_{\mathbf{u}} ; \tilde{\mathbf{p}}_{\mathbf{v}}\right]\right)$.

\subsection{Dataset Specific Design Details}

For most tasks, both the word and edge traversals are done independently and the resultant representations are concatenated as shown above. We tried performing both of these traversals concurrently but it did not give good performance. Some tasks require predicted labels for each node. For these tasks we perform the two traversals in the same way as before but in parallel, and perform classification at each node. Also needed is the computation of an additional representation $(r)$ at each node which is used for the classification with the edge label y (from Section 3.1) as follows,

$$
r= \begin{cases}\tanh \left(\mathbf{W}_{\mathbf{I}}\left(\tilde{\mathbf{p}}_{\mathbf{u}}+\tilde{\mathbf{p}}_{\mathbf{v}}\right)\right), & \text { if current node is an intermediate node } \\ \tanh \left(\mathbf{W}_{\mathbf{L}}\left(\tilde{\mathbf{p}}_{\mathbf{u}}+\mathbf{y}\right)\right), & \text { if current node is a leaf node }\end{cases}
$$

\section{Datasets and Experimental Setup}

In this section, we present the dataset details along with the experimental setup. We first describe our task corpora as well as their statistics in terms of the number of samples: train, test, and validation. Following this, we explain the technical details of our proposed architecture along with its hyper-parameter settings.

Datasets: We wanted to test our model on data from different domains as well as on different tasks. The tasks are: the Stanford Sentiment Treebank (SST) [10] for sentiment analysis, Sentences Involving Compositional Knowledge (SICK) [22] for semantic relatedness $(-\mathrm{R})$ and natural language inference (-E), and the Microsoft Research Paraphrase (MSRP) corpus [23] for paraphrase identification. 
- MSRP: Given a pair of sentences, the task is to identify whether or not they are paraphrases of each other [23]. Train: 4076; Test: 1725; Validation: N/A. We randomly sample and exclude $10 \%$ of the training data and use that as the validation set.

- SICK: The dataset contains sentences derived from video and image annotations. The samples are labelled for both the 3-class SICK-E classification task and the SICK-R regression task which uses real-valued labels between 1 and 5 [22]. We use the same setup as [19] who compute a target distribution $p$ as a function of the predicted score $y$ given by Eqn. 4.1. Train: 4500; Test: 4927; Validation: 500.

$$
\tilde{p_{i}}= \begin{cases}y-\lfloor y\rfloor, & \text { if } i=\lfloor y\rfloor+1 \\ \lfloor y\rfloor-y+1, & \text { if } i=\lfloor y\rfloor \\ 0, & \text { otherwise }\end{cases}
$$

- SST: The samples in this dataset are labelled for both the binary and the 5-class classification task. In this work, we are using only the binary classification labels. Train: 6920; Test: 1821; Validation: 872.

We use accuracy as the standard evaluation metric for SICK-E, MSRP and SST and mean squared error (MSE) along with Pearson's $r$ as the evaluation metric for SICK-R. We use KL-divergence as the loss function for SICK-R and cross entropy as the loss function for the other three tasks.

Experimental Setup: We use GloVe 300-dimensional word vectors [24] to initialize the word embedding layer weights. We use a normal distribution with a cross-validated mean and standard deviation to initialize the edge embedding layer weights. We keep these layers frozen during training. In the multi-head self-attention block, the dimensions of the query, key and value matrices are set to 50 and we use 6 parallel heads on each input. We use a dropout probability of 0.1. During training, the model parameters are updated using the Adagrad algorithm [25] with a fixed learning rate of 0.0002 . We train our model on an Nvidia GeForce GTX 1080Ti GPU and use PyTorch 1.3 for the implementation under the Linux environment. The model that we use for the SICK-E, SICK-R and MSRP tasks has a total of $0.9617 \mathrm{M}$ parameters, whereas the model for the SST task has $0.9927 \mathrm{M}$ parameters.

\section{Results and Discussion}

In this section, we present the effectiveness of equipping a dependency tree transformer with edge label information by evaluating on four NLP tasks. We show that initializing the edge embedding layer weights with a normal distribution having various means and standard deviations impacts the model's performance. We also present how different permutations of the dependency tree edge encoding affect the model's effectiveness. A detailed ablation study reveals the design that led to the best performance. Finally, we conclude this section by visualizing the dependency trees of two test set samples from the SST dataset, one is predicted correctly and the model mistakenly predicts the other.

Table 1 compares our model's performance on four tasks using task-specific evaluation metrics. We report the results for our top performing model having the best initialization settings for edge embedding according to Table 2 and the best configuration of forward and backward edges according to Table 3 . These best performing settings have been arrived at through separate experiments. To do the comparison, we implement both variants of Tree LSTM and Transformer-based architectures and some of the RvNN and LSTM-based models which do not have reported results for all tasks. We only include models that have a comparable number of parameters to ours and do not compare with large language models like BERT [4], BART [5], XLNet [6], and OpenAI-GPT [17]. The evaluation is performed on 
Table 1. Performance comparison of the DT-Transformer+edge label against some state-of-theart LSTM, Transformer and Tree-structured models. Models that we implemented with author provided code are marked with $\dagger$. All models without $\dagger$ (except Ours) have results reported in the original papers. For SICK-R and SST, the number before the slash is what has been reported and the number after the slash is the result of our implementation.

\begin{tabular}{|c|c|c|c|c|c|}
\hline Model Types & Model & $\begin{array}{l}\text { SICK-E } \\
\text { (Acc.\%) }\end{array}$ & $\begin{array}{c}\text { SICK-R } \\
\text { (MSE) }\end{array}$ & $\begin{array}{c}\text { SST } \\
\text { (Acc.\%) }\end{array}$ & $\begin{array}{c}\text { MSRP } \\
\text { (Acc.\%) }\end{array}$ \\
\hline \multirow{5}{*}{ LSTM } & LSTM [19] & 76.80 & .2831 & 84.90 & 71.70 \\
\hline & Bi-LSTM [19] & $82.11 \dagger$ & .2736 & 87.50 & 72.70 \\
\hline & 2-layer LSTM [19] & $78.54 \dagger$ & .2838 & 86.30 & $69.35 \dagger$ \\
\hline & 2-layer Bi-LSTM [19] & $79.66 \dagger$ & .2762 & 87.20 & $70.40 \dagger$ \\
\hline & Infersent [2] & 84.62 & .2732 & 86.00 & 74.46 \\
\hline \multirow{3}{*}{ Transformer } & USE_T [3] & 81.15 & $.5241 \dagger$ & 85.38 & $74.96 \dagger$ \\
\hline & USE_T+DAN [3] & - & - & 86.62 & - \\
\hline & USE_T+CNN [3] & - & - & 86.69 & - \\
\hline \multirow{9}{*}{ Tree Structured } & SDT-RNN [11] & - & .3848 & - & - \\
\hline & RAE [26] & - & - & 82.40 & 76.80 \\
\hline & MV-RNN [27] & $58.14 \dagger$ & - & 82.90 & $66.91 \dagger$ \\
\hline & RNTN [18] & $59.42 \dagger$ & - & 85.40 & $66.91 \dagger$ \\
\hline & DT-RNN [11] & $63.38 \dagger$ & .3822 & 86.60 & $67.51 \dagger$ \\
\hline & DT-LSTM [19] & $83.11 \dagger$ & $.2532 / .2625 \dagger$ & $85.70 / 85.10 \dagger$ & $72.07 \dagger$ \\
\hline & CT-LSTM [19] & $82.00 \dagger$ & $.2734 / .2891 \dagger$ & $88.00 / 87.27 \dagger$ & $70.07 \dagger$ \\
\hline & CT-Transformer [9] & $82.72 \dagger$ & $.3012 \dagger$ & $86.66 \dagger$ & $71.73 \dagger$ \\
\hline & DT-Transformer [9] & $82.95 \dagger$ & $.2774 \dagger$ & $83.12 \dagger$ & $70.34 \dagger$ \\
\hline Ours & DT-Transformer + edge label & 83.32 & .2627 & 83.75 & 71.96 \\
\hline
\end{tabular}

each corpus separately following the standard train/test/validation split instead of assessing on any transfer performance.

First, we wish to highlight the improvement that the edge encodings have provided to our previous DT-Transformer [9]. The incorporation of edge encodings motivated the work presented in this paper. The results in Table 1 indicate that we have been successful in boosting the performance of the DT-Transformer on all four tasks.

Next, we compare our new results with the other model types of interest. On SICKE, our model is achieving $83.32 \%$ accuracy, which is on par with DT-LSTM (83.11\%) as well as CT-LSTM $(82.00 \%)$ and better than our DT-Transformer $(82.95 \%)$ as well as the standard Transformer (81.15\%). On SICK-R, our .2627 MSE is almost the same as the best performed DT-LSTM evaluated in the same settings as ours. On this task, our model is doing significantly better than the standard Transformer (.5241 MSE) and comparatively better than our DT-Transformer having no edge label information. On the SST dataset, our model is getting $83.75 \%$ accuracy which is not as good as most of the tree-structured and sequential models. However, it is doing much better than RAE $(82.40 \%$ Acc.) which is the best performing model on the MSRP dataset. The performance gap between our model and the constituency tree-based models can at least be partially attributable to the fact that these latter ones are trained on more labeled data $(150 K$ vs. $319 K)$. On the MSRP dataset, our model (71.96\% Acc.) is much better than our DT-Transformer $(70.34 \%$ Acc.), our CT-Transformer (71.73\% Acc.) as well as CT-LSTM (70.07\% Acc.) and almost similar to DT-LSTM (72.07\% Acc.). It is to be noted that almost all the sequential models, i.e., Transformer, Infersent, and LSTMs, are doing better compared to the tree-structured models on this task.

Table 2 shows the effect of changing the edge embedding layer weights on the final model performance. We explore this phenomenon by initializing the edge embedding layer weights with a normal distribution having a fixed mean and varying standard deviation. We also experiment with initializing the edge embedding layer weights following the same distribution 
Table 2. Effect of initializing edge embeddings with a fixed mean (0) and changing standard deviation (STD). The last two rows have the respective word embedding mean and STD for SICKE, SICK-R, MSRP, and SST together with the performance when used as the edge embedding mean and STD.

\begin{tabular}{|l|l|c|c|c|c|}
\hline Mean & STD & $\begin{array}{c}\text { SICK-E } \\
\text { (Acc.\%) }\end{array}$ & $\begin{array}{c}\text { SICK-R } \\
\text { (Pearson's r/MSE) }\end{array}$ & $\begin{array}{c}\text { SST } \\
\text { (Acc.\%) }\end{array}$ & $\begin{array}{c}\text { MSRP } \\
\text { (Acc.\%) }\end{array}$ \\
\hline 0 & .025 & 81.65 & $85.89 / .2763$ & 81.22 & 71.57 \\
\hline 0 & .050 & $\mathbf{8 2 . 9 7}$ & $86.02 / .2648$ & 82.32 & 71.79 \\
\hline 0 & .075 & 82.18 & $85.90 / .2684$ & 82.54 & 71.07 \\
\hline 0 & .100 & 81.98 & $85.91 / .2666$ & 81.93 & 71.18 \\
\hline 0 & .125 & 82.24 & $85.95 / .2656$ & 81.37 & 71.18 \\
\hline 0 & .150 & 81.95 & $85.72 / .2698$ & 81.48 & 71.79 \\
\hline 0 & .175 & 82.30 & $85.84 / .2679$ & 81.55 & $\mathbf{7 1 . 9 6}$ \\
\hline 0 & .200 & 81.17 & $\mathbf{8 6 . 1 1} / .2627$ & 81.60 & 71.23 \\
\hline 0 & .225 & 81.79 & $85.81 / .2682$ & $\mathbf{8 3 . 7 5}$ & $\mathbf{7 1 . 9 6}$ \\
\hline 0 & .250 & 82.44 & $85.61 / .2726$ & 81.16 & 71.23 \\
\hline 0 & .275 & 81.79 & $85.94 / .2663$ & 81.49 & 71.35 \\
\hline 0 & .300 & 81.29 & $85.93 / .2663$ & 81.22 & 71.07 \\
\hline 0 & .325 & 81.81 & $85.92 / .2669$ & 81.93 & 70.91 \\
\hline 0 & .350 & 81.65 & $85.34 / .2769$ & 81.11 & 71.40 \\
\hline 0 & .375 & 81.61 & $85.85 / .2693$ & 82.10 & 71.35 \\
\hline 0 & .400 & 82.24 & $85.70 / .2703$ & 82.08 & 71.24 \\
\hline \hline-.0014 & .381 & 81.77 & $85.97 / .2667$ & - & 71.01 \\
\hline-.0066 & .373 & - & & & \\
\hline
\end{tabular}

Table 3. Effect of different ways to form forward and backward edges. $q$ : parent; $x$ : child; $y$ : edge label.

\begin{tabular}{|c|c|c|c|c|c|}
\hline $\begin{array}{c}\text { Forward } \\
\text { Edge }\end{array}$ & $\begin{array}{c}\text { Backward } \\
\text { Edge }\end{array}$ & $\begin{array}{c}\text { SICK-E } \\
\text { (Acc.\%) }\end{array}$ & $\begin{array}{c}\text { SICK-R } \\
\text { (Pearson's r/MSE) }\end{array}$ & $\begin{array}{c}\text { SST } \\
\text { (Acc.\%) }\end{array}$ & $\begin{array}{c}\text { MSRP } \\
\text { (Acc.\%) }\end{array}$ \\
\hline$x y q$ & $x q y$ & 82.97 & $\mathbf{8 6 . 1 1 / . 2 6 2 7}$ & 83.58 & 71.29 \\
\hline$x y q$ & $q y x$ & 81.20 & $85.71 / .2700$ & 82.32 & $\mathbf{7 1 . 9 6}$ \\
\hline$y x q$ & $y q x$ & 81.12 & $85.48 / .2741$ & 82.10 & 70.63 \\
\hline$q y x$ & $y q x$ & 81.14 & $85.37 / .2786$ & $\mathbf{8 3 . 7 5}$ & 68.57 \\
\hline$q y x$ & $\times$ & $\mathbf{8 3 . 3 2}$ & $85.31 / .2781$ & 79.68 & 69.35 \\
\hline$q x y$ & $\times$ & 81.65 & $84.90 / .2848$ & 81.65 & 69.02 \\
\hline$x y q$ & $\times$ & 81.71 & $85.69 / .2702$ & 82.92 & 71.68 \\
\hline$x q y$ & $\times$ & 82.10 & $84.91 / .2839$ & 81.93 & 71.79 \\
\hline$y q x$ & $\times$ & 81.33 & $85.13 / .2825$ & 81.11 & 68.07 \\
\hline$y x q$ & $\times$ & 81.81 & $85.12 / .2819$ & 82.32 & 69.29 \\
\hline
\end{tabular}

as the word embedding layer. As can be seen, the best performances across the datasets are achieved with mean 0 and standard deviation in the range of $0.175-0.225$, except for SICK-E. The best performance is with standard deviation 0.05. Similar to the word embedding layer weights, we keep the edge embedding layer weights frozen by not including them in the training.

Table 3 shows the effect of different ways to encode forward and backward edges on the final model performance. Together, there are $3 !+(3 ! \times 3 !)$ ways to encode forward and backward edges, but here we only report some of the noteworthy results as the others are giving similar or comparatively poorer performance. We do not experiment with only backward edges as it seemed similar to having forward edges only. Of our four tasks, having both forward and backward edges together gives better performance as opposed to having them alone. As can be seen, there is no fixed pattern for encoding the edges. Instead, it is dataset specific and can only be determined through a brute force experiment. 


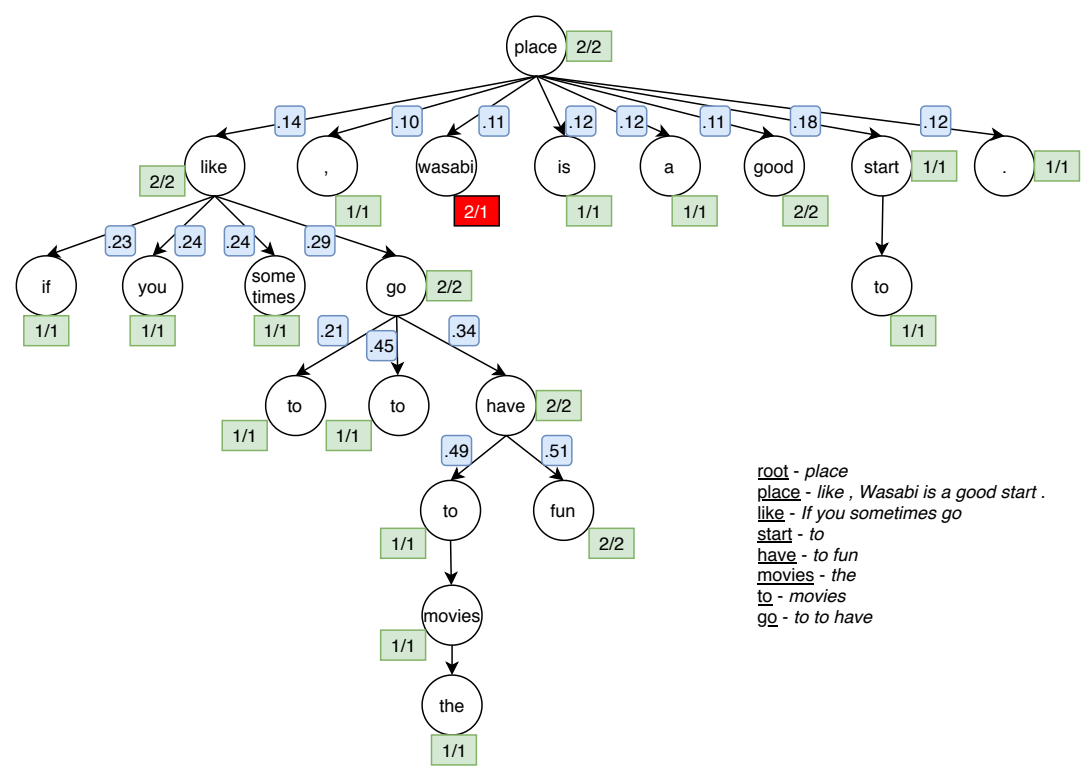

(a) If you sometimes like to go to the movies to have fun, Wasabi is a good place to start.

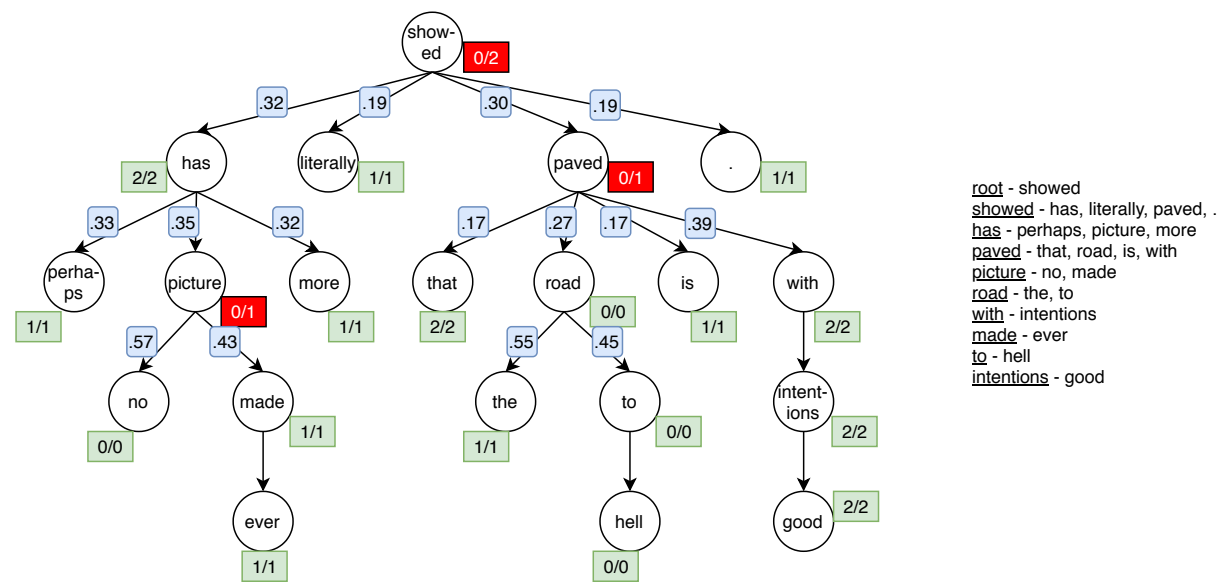

(b) Perhaps no picture ever made has more literally showed that the road to hell is paved with good intentions .

Figure 3. Attentive tree visualization (DT-Transformer + edge label). 0-Negative; 1Neutral; 2-Positive. X/Y: X-Predicted label; Y-True label. The blue squares indicate attention probabilities.

Figure 3 shows how the model puts attention on different parts of a dependency tree and computes the final sentiment. The visualization also shows whether the model is making a correct decision or not on each of the nodes. As can be seen in Figure 3(a), our model gets the final sentiment of the sentence correctly and it also correctly classifies the sentiment of each of the nodes except the word "wasabi". At the first level, the word "like" is getting $14 \%$ attention which gets its positive sentiment from the subtree $g o \rightarrow t o$, to, have which itself is getting highest attention (29\%) among its siblings. Also, in the subtree have $\rightarrow$ to, fun, the edge "fun" is getting a comparatively higher probability which is a positive sentiment word. In the second example shown in Figure 3(b), our model is making correct predictions for almost all nodes but getting some of the key ones wrong. The sentiment of the subtree 
Table 4. Ablation study. (Default settings: word_embedding_train = False, edge_embedding_train $=$ False, pooling_type $=$ maxpool, word_attention $=$ multi-head, edge_attention $=$ multi-head, optimizer $=$ Adagrad, $f c \_l a y e r=$ non-linear, word-edge_projection $=$ not-shared, forward_edge $=$ True, backward_edge $=$ True, projection_residual $=$ False, final_activation $=\log$-softmax)

\begin{tabular}{|c|c|}
\hline \multicolumn{2}{|l|}{ SICK-E } \\
\hline Model Settings & Accuracy (\%) \\
\hline fc_layer $=$ linear & 82.97 \\
\hline fc_layer $=$ linear, final_activation $=$ softmax & 80.55 \\
\hline backward_edge $=$ False & 81.88 \\
\hline backward_edge $=$ False, $f c \_$layer $=$linear & 82.87 \\
\hline $\begin{array}{l}\text { backward_edge }=\text { False }, \text { word_attention }=\text { multi-branch } \\
\text { edge_attention }=\text { multi-branch }, \text { fc_layer }=\text { linear }\end{array}$ & 83.32 \\
\hline \multicolumn{2}{|l|}{ SICK-R } \\
\hline Model Settings & Pearson's r/MSE \\
\hline Default & $86.11 / .2627$ \\
\hline pooling_type $=$ mean & $82.21 / .3451$ \\
\hline word_attention $=$ multi-branch & $82.21 / .3451$ \\
\hline word_attention $=$ single-head, edge_attention $=$ single-head & $81.00 / .3507$ \\
\hline word_attention $=$ multi-branch, edge_attention $=$ multi-branch & $84.83 / .2872$ \\
\hline \multicolumn{2}{|l|}{ SST } \\
\hline Model Settings & Accuracy (\%) \\
\hline Default + word traversal to classify both leaf and intermediate nodes & 80.89 \\
\hline Default + word and edge traversal to classify both leaf and intermediate nodes & 81.60 \\
\hline $\begin{array}{l}\text { Default }+ \text { word traversal to classify leaf, word and edge traversal to classify } \\
\text { intermediate nodes }\end{array}$ & 81.99 \\
\hline Default + word and edge traversal to classify both leaf and intermediate nodes & 83.75 \\
\hline Default + word and edge traversal to classify both leaf and intermediate nodes & 82.70 \\
\hline \multicolumn{2}{|l|}{ MSRP } \\
\hline Model Settings & Accuracy (\%) \\
\hline Default & 71.96 \\
\hline projection_residual $=$ True & 71.07 \\
\hline optimizer $=$ SGD, backward_edge $=$ False & 71.18 \\
\hline word_attention $=$ single-head, edge_attention $=$ single-head & 70.74 \\
\hline word_attention $=$ multi-branch, edge_attention & 70.68 \\
\hline
\end{tabular}

paved $\rightarrow$ that, road, is, with is predicted as negative, which propagates and makes the final decision wrong. However, the misprediction in subtree picture $\rightarrow$ no, made does not have any effect because this entire subtree is getting almost equal attention as its siblings. Overall, having these attention probabilities provides transparency to the model and tells how it is thinking about a specific example and helps us to relate to how we as a human would treat it. We have not visualized the output for SICK and MSRP datasets since the attention weights were equal or almost equal, respectively. The reasons could be the very short sentences in the SICK dataset and the syntactic similarity between the two sentences in the SICK and MSRP datasets.

\subsection{Ablation Study}

We also perform an ablation study on our model by plugging in and out different modules. The choice of modules that we have studied is motivated by various studies. Regarding attention, investigations have shown that the choice of single- and multi-head and multibranch attention is possibly domain and task dependent [28, 29]. The training or freezing of word embeddings is a common features of ablation studies (e.g., [30, 31]). This prompts 
us to also consider the training and freezing of the edge embeddings. The other ablation features are commonly tested.

The SST task is different from the others in that each node in the tree is given a sentiment value rather than a single score being given to each sample in the dataset. We first differentiate intermediate and leaf nodes. The ablation study investigates the turning on and off of the word and edge traversal modules for these two classes of nodes.

We report the results in Table 4 . We show only the 5 best performing settings on each dataset. For better readability, we provide default settings and then override them by turning on and off different modules and changing their values as well. As can be seen, the default settings are giving the best performance only for the SICK-R and MSRP datasets. For SICK-E, forward edges only (see Table 3), multi-branch attention as the composition function, and a linear classifier gives the best performance. For the SST dataset, we use the default settings for the tree traversal and the settings defined in Eqn. 3.10 for the node-wise classification.

\section{Conclusion}

In this paper, we propose a novel way to include edge label information inside a dependency tree transformer which encodes natural language grammar trees utilizing the modules designed for the standard Transformer. We show that having edge label information definitely helps and gives a boost compared to not having it. We infer that having only a good edge encoding with a proper design principle is enough to obtain the observed improvement and does not require training of this encoding. We also provide an ablation study showing the roadmap of achieving an optimal performance through the use of different modules and design settings. Our study shows that having edge label information complements a dependency tree transformer by putting it on par with Tree LSTMs and making it even better compared to the standard Transformer.

\section{Acknowledgements}

This research is partially funded by The Natural Sciences and Engineering Research Council of Canada (NSERC) through a Discovery Grant to Robert E. Mercer. We also acknowledge the helpful comments provided by the reviewers.

\section{References}

[1] T. Mikolov, I. Sutskever, K. Chen, G. S. Corrado, and J. Dean. "Distributed representations of words and phrases and their compositionality". In: Advances in Neural Information Processing Systems. 2013, pp. 3111-3119.

[2] A. Conneau, D. Kiela, H. Schwenk, L. Barrault, and A. Bordes. "Supervised Learning of Universal Sentence Representations from Natural Language Inference Data". In: Proceedings of the 2017 Conference on Empirical Methods in Natural Language Processing. 2017, pp. 670680 .

[3] D. Cer, Y. Yang, S.-y. Kong, N. Hua, N. Limtiacob, R. S. John, N. Constant, M. GuajardoCéspedes, S. Yuanc, C. Tar, Y.-H. Sung, B. Strope, and R. Kurzweil. "Universal Sentence Encoder". In: arXiv preprint arXiv:1803.11175 (2018).

[4] J. Devlin, M.-W. Chang, K. Lee, and K. Toutanova. "BERT: Pre-training of deep bidirectional transformers for language understanding". In: arXiv preprint arXiv:1810.04805 (2018).

[5] M. Lewis, Y. Liu, N. Goyal, M. Ghazvininejad, A. Mohamed, O. Levy, V. Stoyanov, and L. Zettlemoyer. "BART: Denoising sequence-to-sequence pre-training for natural language generation, translation, and comprehension". In: arXiv preprint arXiv:1910.13461 (2019). 
[6] Z. Yang, Z. Dai, Y. Yang, J. Carbonell, R. R. Salakhutdinov, and Q. V. Le. "XLNnet: Generalized autoregressive pretraining for language understanding". In: Advances in Neural Information Processing Systems. 2019, pp. 5753-5763.

[7] M.-C. De Marneffe and C. D. Manning. Stanford typed dependencies manual. Tech. rep. Stanford University, 2008.

[8] D. M. Cer, M.-C. De Marneffe, D. Jurafsky, and C. D. Manning. "Parsing to Stanford Dependencies: Trade-offs between Speed and Accuracy." In: Proceedings of the Seventh International Conference on Language Resources and Evaluation (LREC'10). 2010, pp. 1628-1632.

[9] M. Ahmed, M. R. Samee, and R. E. Mercer. "You Only Need Attention to Traverse Trees". In: Proceedings of the 57th Annual Meeting of the Association for Computational Linguistics. 2019, pp. 316-322.

[10] R. Socher, C. C.-Y. Lin, A. Y. Ng, and C. D. Manning. "Parsing Natural Scenes and Natural Language with Recursive Neural Networks". In: Proceedings of the 28th International Conference on International Conference on Machine Learning (ICML'11). Bellevue, Washington, USA, 2011, pp. 129-136. ISBN: 978-1-4503-0619-5.

[11] R. Socher, A. Karpathy, Q. V. Le, C. D. Manning, and A. Y. Ng. "Grounded Compositional Semantics for Finding and Describing Images with Sentences". In: Transactions of the Association for Computational Linguistics 2 (2014), pp. 207-218.

[12] Z. Yang, D. Yang, C. Dyer, X. He, A. Smola, and E. Hovy. "Hierarchical Attention Networks for Document Classification". In: Proceedings of the 2016 Conference of the North American Chapter of the Association for Computational Linguistics: Human Language Technologies. 2016, pp. 1480-1489.

[13] A. Kumar, O. Irsoy, P. Ondruska, M. Iyyer, J. Bradbury, I. Gulrajani, V. Zhong, R. Paulus, and R. Socher. "Ask me anything: Dynamic memory networks for natural language processing". In: International Conference on Machine Learning. 2016, pp. 1378-1387.

[14] A. Parikh, O. Täckström, D. Das, and J. Uszkoreit. "A Decomposable Attention Model for Natural Language Inference". In: Proceedings of the 2016 Conference on Empirical Methods in Natural Language Processing. Austin, Texas, 2016, pp. 2249-2255.

[15] A. Vaswani, N. Shazeer, N. Parmar, J. Uszkoreit, L. Jones, A. N. Gomez, Ł. Kaiser, and I. Polosukhin. "Attention is all you need". In: Advances in Neural Information Processing Systems. 2017, pp. 5998-6008.

[16] K. Ahmed, N. S. Keskar, and R. Socher. "Weighted transformer network for machine translation". In: arXiv preprint arXiv:1711.02132 (2017).

[17] A. Radford, K. Narasimhan, T. Salimans, and I. Sutskever. Improving language understanding with unsupervised learning. Tech. rep. OpenAI, 2018.

[18] R. Socher, A. Perelygin, J. Wu, J. Chuang, C. D. Manning, A. Ng, and C. Potts. "Recursive Deep Models for Semantic Compositionality Over a Sentiment Treebank". In: Proceedings of the 2013 Conference on Empirical Methods in Natural Language Processing. 2013, pp. 16311642 .

[19] K. S. Tai, R. Socher, and C. D. Manning. "Improved Semantic Representations From TreeStructured Long Short-Term Memory Networks". In: Proceedings of the 53rd Annual Meeting of the Association for Computational Linguistics and the 7th International Joint Conference on Natural Language Processing (Volume 1: Long Papers). Beijing, China, 2015, pp. 15561566.

[20] M. Ahmed, M. R. Samee, and R. E. Mercer. "Improving tree-LSTM with tree attention". In: 2019 IEEE 13th International Conference on Semantic Computing (ICSC). 2019, pp. 247254.

[21] C. D. Manning, M. Surdeanu, J. Bauer, J. Finkel, S. J. Bethard, and D. McClosky. "The Stanford CoreNLP Natural Language Processing Toolkit". In: Association for Computational Linguistics (ACL) System Demonstrations. 2014, pp. 55-60.

[22] M. Marelli, L. Bentivogli, M. Baroni, R. Bernardi, S. Menini, and R. Zamparelli. "SemEval2014 Task 1: Evaluation of compositional distributional semantic models on full sentences through semantic relatedness and textual entailment". In: Proceedings of the 8th International Workshop on Semantic Evaluation (SemEval 2014). 2014, pp. 1-8. 
[23] B. Dolan, C. Quirk, and C. Brockett. "Unsupervised construction of large paraphrase corpora: Exploiting massively parallel news sources". In: Proceedings of the 20th International Conference on Computational Linguistics. 2004, pp. 350-356.

[24] J. Pennington, R. Socher, and C. Manning. "GloVe: Global vectors for word representation". In: Proceedings of the 2014 Conference on Empirical Methods in Natural Language Processing (EMNLP). 2014, pp. 1532-1543.

[25] J. Duchi, E. Hazan, and Y. Singer. "Adaptive subgradient methods for online learning and stochastic optimization". In: J. of Machine Learning Research 12.61 (2011), pp. 2121-2159.

[26] R. Socher, E. H. Huang, J. Pennington, C. D. Manning, and A. Y. Ng. "Dynamic pooling and unfolding recursive autoencoders for paraphrase detection". In: Advances in Neural Information Processing Systems. 2011, pp. 801-809.

[27] R. Socher, B. Huval, C. D. Manning, and A. Y. Ng. "Semantic compositionality through recursive matrix-vector spaces". In: Proceedings of the 2012 Joint Conference on Empirical Methods in Natural Language Processing and Computational Natural Language Learning. 2012, pp. 1201-1211.

[28] P. Michel, O. Levy, and G. Neubig. "Are sixteen heads really better than one?" In: Advances in Neural Information Processing Systems. Vol. 32. 2019.

[29] M. Ahmed and R. E. Mercer. "Efficient transformer-based sentence encoding for sentence pair modelling". In: Canadian Conference on Artificial Intelligence. Springer. 2019, pp. 146-159.

[30] T. Q. Nguyen and D. Chiang. "Transfer learning across low-resource, related languages for neural machine translation". In: Proceedings of the Eighth International Joint Conference on Natural Language Processing (Volume 2: Short Papers). 2017, pp. 296-301.

[31] A. K. Lampinen and J. L. McClelland. "One-shot and few-shot learning of word embeddings". In: arXiv preprint arXiv:1710.10280 (2017). 